\title{
Synthesis of nanoparticulate anatase and rutile crystallites at low temperatures in the Pluronic F127 microemulsion system
}

\author{
Erik Nilsson ${ }^{\text {a) }}$ \\ Applied Surface Chemistry, Dept. of Chemical and Biological Engineering, Chalmers University of Technology, \\ SE 41296 Göteborg, Sweden \\ Hirotoshi Furusho \\ Structural Chemistry, Department of Materials and Environmental Chemistry, Stockholm University, SE 10691 \\ Stockholm, Sweden \\ Osamu Terasaki \\ Structural Chemistry, Department of Materials and Environmental Chemistry, Stockholm University, SE 10691 \\ Stockholm, Sweden; and Graduate School of EEWS (WCU), KAIST, Daejeon 305-701, Republic of Korea \\ Anders E.C. Palmqvist ${ }^{\text {b) }}$ \\ Applied Surface Chemistry, Dept. of Chemical and Biological Engineering, Chalmers University of Technology, SE \\ 41296 Göteborg, Sweden
}

(Received 1 May 2010; accepted 7 September 2010)

\begin{abstract}
A low-temperature synthesis method for preparing nanosized $\mathrm{TiO}_{2}$ crystallites has been developed based on a Pluronic F127 microemulsion system. Both anatase and rutile polymorphs can be prepared, and there exists a temperature window between 40 and $50{ }^{\circ} \mathrm{C}$ where the formation of rutile is favored over anatase. At $60{ }^{\circ} \mathrm{C}$ and above, anatase is kinetically favored and only very slowly transforms to rutile at $60{ }^{\circ} \mathrm{C}$. The results differ from previous observations regarding formation kinetics and temperature range for rutile formation as well as in the microscopic aggregation of the formed nanoparticles. This development of a low-temperature synthesis of crystalline titania nanoparticles within the Pluronic block copolymer system is an important and enabling step toward devising a direct synthesis route for the formation of ordered mesoporous and crystalline titania.
\end{abstract}

\section{INTRODUCTION}

Titania $\left(\mathrm{TiO}_{2}\right)$ is a photoactive compound of great importance for photophysical and photochemical applications. In 1972, Fujishima and Honda showed that $\mathrm{TiO}_{2}$ can be used to photocatalytically split water to hydrogen and oxygen using solar light, a topic of great current societal interest and intense research. ${ }^{1}$ The strong photocatalytic oxidation capacity of $\mathrm{TiO}_{2}$ is also used for degradation of organic contaminants in water and air. $^{2}$ In this area, selfcleaning coatings of titania are saving large amounts of energy. ${ }^{3}$ More recently, titania has been increasingly studied for its great potential as an active component in solar cells as demonstrated by Oregan and Grätzel. ${ }^{4}$ Titania occurs as a common compound in the earth's crust where its three most common polymorphs are brookite, anatase, and rutile. Rutile is thermodynamically stable, whereas anatase and brookite are metastable. The polymorphs have somewhat different electronic and optical properties and hence different areas of application. Anatase has been reported to exhibit higher photocatalytic activity than rutile. ${ }^{2,5}$ However, there are studies that show

Address all correspondence to these authors.

a)e-mail: erik.nilsson@chalmers.se

b) e-mail: adde@ chalmers.se

DOI: $10.1557 /$ jmr.2010.5 that rutile nanoparticles synthesized at low temperatures show similar activity but may catalyze different reaction paths compared with anatase. ${ }^{6-8}$

A way of further improving the effectiveness of crystalline titania for photocatalytic oxidation is to prepare it in a mesoporous form. ${ }^{9}$ A similar benefit has been shown for dye-sensitized solar cells. ${ }^{10}$ Mesoporous materials have pores in the range of 2 to $50 \mathrm{~nm}$ and large specific surface areas, the combination of which increases the amount of active sites and the accessibility to these sites for an electrolyte or reactant mixture. Ordered mesoporous metal oxides were first reported in the early 1990s. ${ }^{11,12}$ They are typically made by removal of the organic structure-directing molecules used in the formation of a mesostructured organic/inorganic composite. The mesostructured composite is prepared under wet chemical conditions using amphiphilic molecules, as structuredirecting agents. A large number of ordered mesoporous materials have been prepared using this methodology. ${ }^{13-17}$ However, the walls of an as-prepared ordered mesoporous material are most often amorphous since crystallization usually requires temperatures that exceed those available under the aqueous conditions used. This is the case also for titania, and the as-prepared mesostructured composite is typically amorphous. ${ }^{18}$ Crystallization of the titania may subsequently be affected through solid-state conversion of 
the amorphous composite into crystalline nanoparticles organized in a mesoporous structure. However, such heat treatment often induces loss of meso-order because of thermal instability as a result of the crystallization and the crystal growth, and rutile especially remains a challenge to prepare in an ordered mesoporous state. One attractive way to overcome these obstacles would be to prepare nanoparticles of crystalline titania and subsequently induce a co-assembly process of the nanocrystals with a liquid crystal-forming amphiphilic template. This requires a system that allows for crystallization of nanoparticulate titania at low temperatures and which is also able to form liquid crystalline phases.

A number of different synthesis technologies are being developed for preparation of nanoparticulate materials including high-temperature gas synthesis methods such as flame synthesis and plasma processes, ${ }^{19,20}$ supercritical methods, ${ }^{21}$ and low-temperature solution methods such as sol-gel, solvothermal, or microemulsion. In general, the solution-based methods are slower but compatible with softer chemical interactions present in surfactant selfassembly methodologies. Such methods have shown it possible to prepare high-temperature stable oxide phases at low temperatures. Exploiting the faster dynamics and the large interfacial area between oil and water in microemulsions allows for increased reaction rates and the use of hydrophobic molecular precursors under mild conditions. Hence, rutile was recently prepared with very high specific surface area at room temperature through a microemulsion-mediated route using a system with the nonionic surfactant Triton $\mathrm{X}-100 .{ }^{22}$ The topic of this work is to develop a similar synthesis route for anatase and rutile using a microemulsion system based on the nonionic block copolymer Pluronic F127. ${ }^{23}$ This system is especially interesting because Pluronic polymers display phase diagrams containing a plethora of liquid crystalline phases that have successfully been used for formation of mesoordered hybrid materials and, upon subsequent removal of the polymer, of mesoporous metal oxides. ${ }^{16}$

\section{EXPERIMENTAL METHODS}

A series of syntheses was performed with synthesis mixture compositions positioned within the reversed microemulsion area $\left(\mathrm{L}_{2}\right)$ of the ternary phase diagram between Pluronic F127, butanol and water. ${ }^{23}$ The butanol that is formed upon hydrolysis of the titania precursor shifts the position of the composition in the phase diagram only to a minimal extent and only within the boundaries of the reversed microemulsion phase. The chemicals used in this study were Pluronic F127 $\left(\mathrm{EO}_{100} \mathrm{PO}_{70} \mathrm{EO}_{100}\right.$, Sigma), titanium(IV)butoxide (97\%, Aldrich), 1-butanol ( $\geq 99.4 \%$, Sigma-Aldrich), hydrochloric acid (37\%, SigmaAldrich), and nitric acid (69\%, Sigma-Aldrich). The weight ratio of $\mathrm{Ti}(\mathrm{IV})$ butoxide/diluted acid was equal to 0.85 for all syntheses. Ti(IV)butoxide was added dropwise to the microemulsion under continuous stirring. The mixture was put in a polypropylene bottle or a teflon-lined stainless steel autoclave at specified temperature and time. The formed products were separated from the synthesis solution by centrifugation and washed in $\mathrm{CH}_{2} \mathrm{Cl}_{2}$ to remove the surfactant. Finally, the $\mathrm{CH}_{2} \mathrm{Cl}_{2}$ was separated from the product by centrifugation, and the samples were subsequently dried at room temperature in open air overnight. Powder x-ray diffraction measurements were performed on a Bruker D8 advance diffractometer using $\mathrm{Cu} \mathrm{K} \alpha$ radiation $(\lambda=1.54 \AA)$. The mean crystallite size was calculated according to the Scherrer equation based on the Bragg peak width at half maximum intensity. ${ }^{24,25}$ Transmission electron microscopy (TEM) observation was performed on a JEOL JEM-2100F with $C_{\mathrm{s}}=0.5 \mathrm{~mm}$ operated at $200 \mathrm{kV}$. The samples were dispersed in ethanol and dropped onto a microgrid, and images were recorded with a Gatan Ultrascan CCD camera. For the SEM observation, the samples were dropped on graphite. SEM images were acquired with a JEOL JSM-7401F. The accelerating and retarding voltages used were 3 and $2 \mathrm{kV}$, respectively; therefore, the net electron impact voltage onto the sample was $1 \mathrm{kV}$ using gentle-beam method. Nitrogen gas adsorption measurements were performed at $77 \mathrm{~K}$ on a Micromeretic TriStar instrument, where prior to measurement, the samples were exposed to vacuum for $3 \mathrm{~h}$ at $100{ }^{\circ} \mathrm{C}$ to remove moisture. The specific surface area was measured according to the Brunauer-Emmett-Teller (BET) method. ${ }^{26}$

\section{RESULTS AND DISCUSSION}

At first, the influence of the microemulsion composition on the formation of $\mathrm{TiO}_{2}$ nanoparticles was established by performing a series of syntheses at $50{ }^{\circ} \mathrm{C}$ at the different positions in the $\mathrm{L}_{2}$ phase region presented in Fig. 1. Composition $A$ is centrally positioned within the $L_{2}$ phase, whereas compositions $\mathrm{B}$ to $\mathrm{G}$ are positioned closer to the boundaries of the $\mathrm{L}_{2}$ phase to evaluate the extreme compositions of the microemulsion. Apparently the composition has a great impact on the crystallization of $\mathrm{TiO}_{2}$, and syntheses A, B, and G clearly show diffraction peaks corresponding to rutile (see supporting information). The crystallinity is very low or absent for syntheses made with low water content, i.e., compositions $\mathrm{C}$ to E. For syntheses made in compositions $\mathrm{C}$ and $\mathrm{D}$, weak peaks at $2 \theta=36^{\circ}$ and $55^{\circ}$ can be discerned indicating presence of some rutile. Clearly, composition A was most favorable for the formation of crystalline $\mathrm{TiO}_{2}$ of the compositions evaluated, and the study was henceforth focused on this point.

The three additional experimental parameterstemperature, synthesis time, and type of acid-were evaluated one at a time for syntheses performed with composition A. Figure 2 shows the $\mathrm{x}$-ray diffractograms of the products obtained after $96 \mathrm{~h}$ in a series of runs performed 
at different temperatures and using $5 \mathrm{M} \mathrm{HCl}$ as acid solution. In contrast to results reported for the Triton X100 system, ${ }^{22}$ no crystallization occurred at room temperature where the material formed was amorphous even after 4 days of reaction. At synthesis temperatures of 40 and $50{ }^{\circ} \mathrm{C}$ rutile was formed, whereas if the synthesis solution was instead hydrothermally treated at $120{ }^{\circ} \mathrm{C}$ only anatase was formed in agreement with the Triton X-100 system. However, a prolonged synthesis at $120{ }^{\circ} \mathrm{C}$ did not result in a transition to rutile for the Pluronic system in contrast to the Triton X-100 system for which anatase was converted into

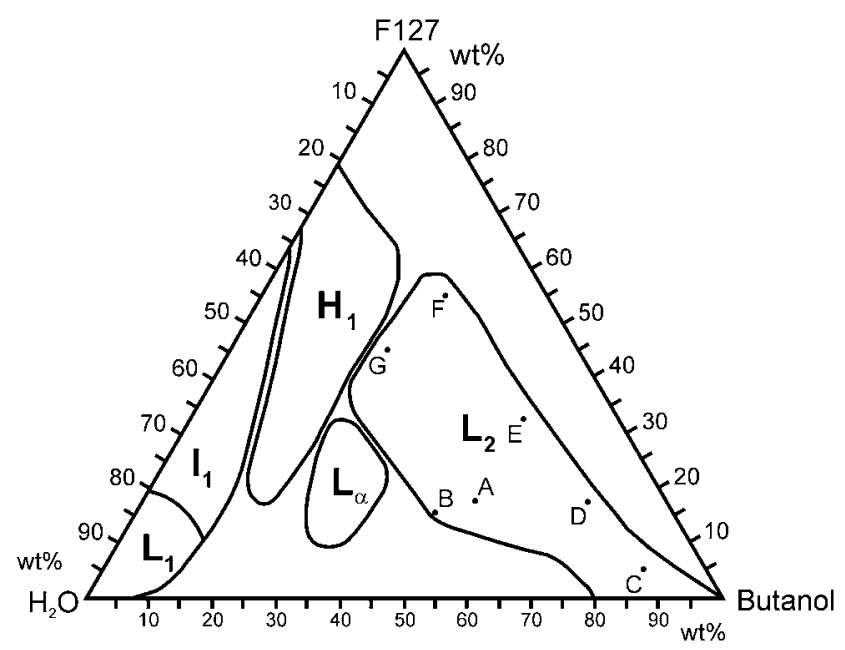

FIG. 1. Phase diagram of $\mathrm{H}_{2} \mathrm{O} /$ butanol/F127 redrawn from Holmqvist et al. ${ }^{23}$ with compositions of microemulsions used in the synthesis of $\mathrm{TiO}_{2}$ marked with letters $\mathrm{A}-\mathrm{G}$ within the $\mathrm{L}_{2}$ phase region.

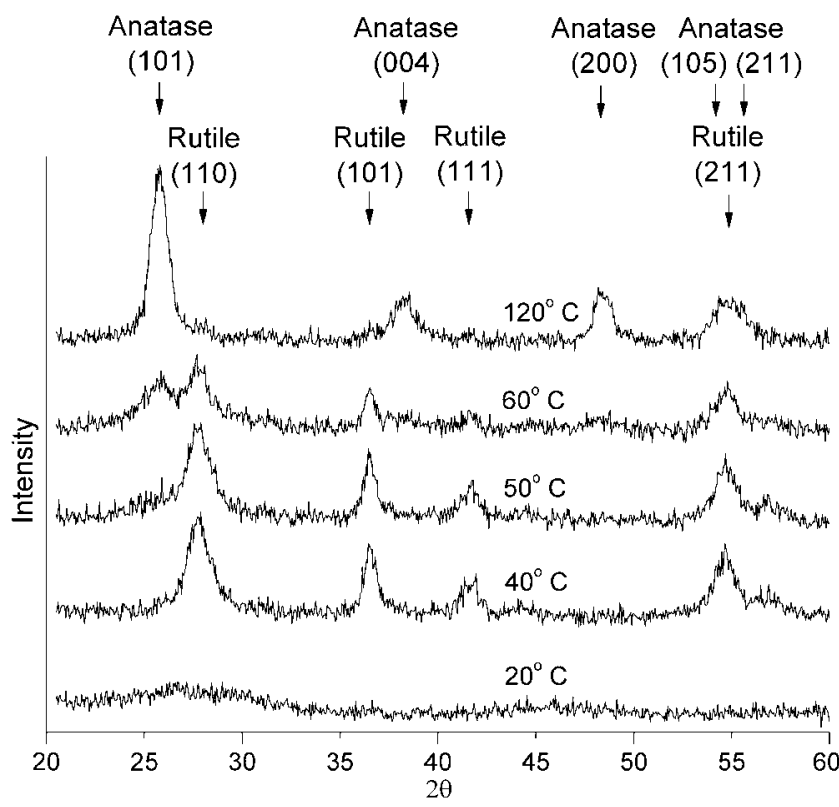

FIG. 2. Powder $\mathrm{x}$-ray diffractograms of products formed during $96 \mathrm{~h}$ of synthesis, as a function of synthesis temperature, using a microemulsion with composition $\mathrm{A}$ and $5 \mathrm{M} \mathrm{HCl}$ as acid solution. rutile after roughly $12 \mathrm{~h}$ at $120^{\circ} \mathrm{C} .{ }^{22} \mathrm{We}$ note, however, that there seems to be a discrepancy between the kinetics of the conversion from anatase to rutile at $120{ }^{\circ} \mathrm{C}$ for the Triton $\mathrm{X}-100$ system, apparently due to differences in autoclave type used. ${ }^{8,22}$

The two amphiphiles Pluronic F127 and Triton X-100 are both nonionic. Pluronic F127 is a triblock copolymer built up by a central, 70 unit long, polypropyleneoxide chain and two, 100 unit long, polyethyleneoxide chains. In solution, F-127 will form a hydrophilic headgroup by coiling up its two polyethyleneoxide chains, while its hydrophobic chain is folded out in the oil matrix. The Triton X-100 surfactant is a much smaller molecule built up by a hydrophilic polyethyleneoxide chain of 10 units and a hydrophobic chain consisting of a 4-(1,1,3, 3-tetramethylbutyl)-phenyl group. These large differences in both size and composition of the two amphiphiles are expected to affect the formation kinetics as well as the colloidal stability and propensity for aggregation of the formed nanoparticles as further discussed below.

Using a synthesis temperature of $60{ }^{\circ} \mathrm{C}$, both rutile and anatase were formed for the Pluronic F127 system. Figure 3 shows the x-ray diffractograms of products formed at $60{ }^{\circ} \mathrm{C}$ as a function of synthesis time. Clearly, the composition of the product gradually changes toward more rutile at this temperature. The corresponding $\mathrm{x}$-ray diffractograms for syntheses made at $50{ }^{\circ} \mathrm{C}$ show that rutile exists already after $8 \mathrm{~h}$ of treatment of the synthesis solution, and as expected it is the only polymorph present also for the longer time periods studied (see supporting information). The influence of the hydrochloric acid concentration is shown in Fig. 4, and it was found not to affect the crystal structure of the formed $\mathrm{TiO}_{2}$ to a large extent, although a small shoulder for the anatase (101) reflection is discerned in the $\mathrm{x}$-ray diffractogram when

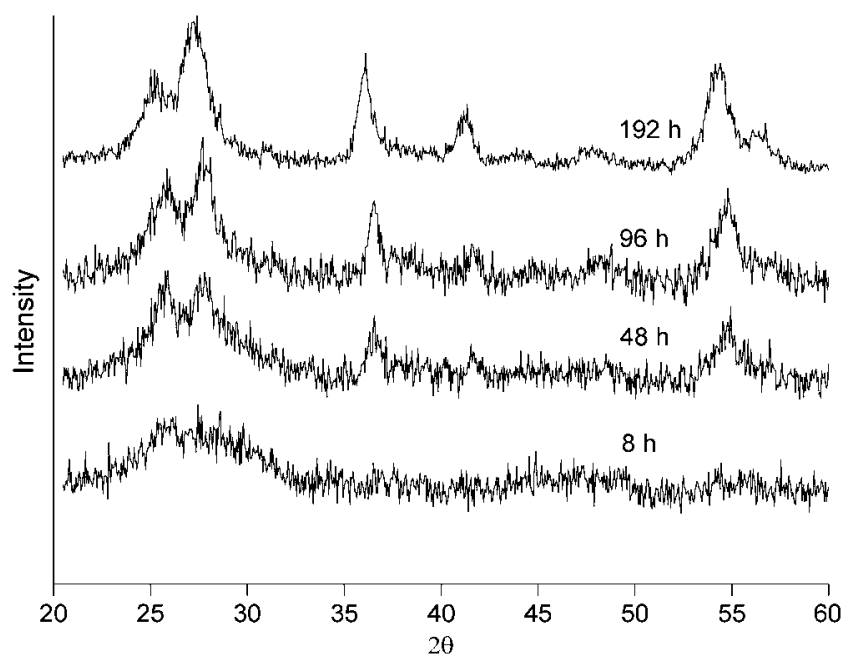

FIG. 3. Powder x-ray diffractograms of materials formed at $60{ }^{\circ} \mathrm{C}$ of synthesis, as a function of synthesis time, using a microemulsion with composition $\mathrm{A}$ and $5 \mathrm{M} \mathrm{HCl}$ as acid solution. 


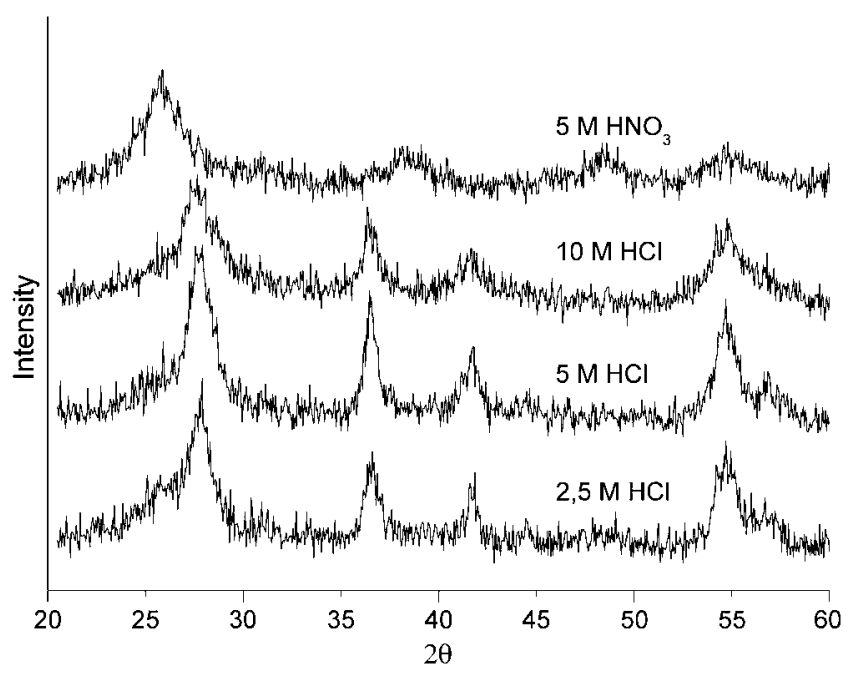

FIG. 4. Powder $\mathrm{x}$-ray diffractograms of products obtained at $50{ }^{\circ} \mathrm{C}$ after $96 \mathrm{~h}$ for syntheses carried out in a microemulsion with composition A using different acid solutions.

$2.5 \mathrm{M} \mathrm{HCl}$ was used. In contrast, when using $5 \mathrm{M} \mathrm{HNO}_{3}$ instead of $\mathrm{HCl}$, anatase is the only polymorph formed. A similar dependence on acid type was reported also for the Triton X-100 system. ${ }^{8}$

In general, the formation of crystalline nanoparticles is governed by the interplay between thermodynamics and formation kinetics. Under the conditions present in microemulsion systems, the relative rates of nucleation and growth of the different polymorphs are what determines which polymorph forms and also what frequently facilitates less thermodynamically favored phases to form. It is the combination of reaction mixture molar composition and temperature used that governs the rates of formation, which may have different temperature dependencies due to differences in their rate-determining activation energies. This complexity is what facilitates the low-temperature synthesis of high-temperature phases such as rutile and also enables kinetic control over thermodynamic driving force, which allows for anatase to be formed before rutile.

Large differences between microemulsion systems such as types of amphiphile and acid anion used should be expected to induce large effects on the formation kinetics of the nanoparticles as well as on their thermodynamic properties because of their large surface-to-bulk ratio. The complexity of these syntheses may therefore lead to apparently contradictory results when comparing different microemulsion systems because they show different dependencies to a certain variable. In the present study, the rates of formation of the two polymorphs anatase and rutile are shown to be affected by a number of synthesis variables, such as, e.g., temperature and molar composition used in the reaction solution.

To rationalize the effect of the acid used, the polymorph-directing properties of the anion of the acid used is apparent. It affects the relative rates of formation of the two $\mathrm{TiO}_{2}$ polymorphs as evidenced by the fact that both polymorphs can be formed at the same temperature using different anions, and because one of the anions can induce formation of both polymorphs by proper choice of temperature. These observations are in accord with a formation mechanism in which the acid anion affects the relative rates of formation of the two polymorphs and where the polymorphs have different rate-determining activation energies and thus different temperature dependencies. The most likely molecular explanation for this is that the anions participate in the nucleation and growth of the $\mathrm{TiO}_{2}$ particles presumably through different modes of coordination at the growing surfaces of the particles. This is indeed to be expected as the two anions used (nitrate and chloride) exhibit largely different coordination characteristics in aqueous solutions. The differences in anion dependence between the two microemulsion systems compared here may be understood by realizing that it is the combination of amphiphile and anion that is decisive in the governing energies involved in the nucleation and growth of the nanoparticles rather than the individual components used.

To compare the microscopic structure of the formed particles and their aggregation characteristics between samples prepared in the Pluronic F127 system with those prepared in the Triton X-100 system, a series of TEM and SEM images were collected for two samples. In Fig. 5, TEM micrographs of a sample formed during $8 \mathrm{~h}$ of synthesis at $40{ }^{\circ} \mathrm{C}$ using a microemulsion with composition $\mathrm{A}$ and $5 \mathrm{M} \mathrm{HCl}$ as acid solution are compared with micrographs of a sample prepared with a microemulsion based on Triton X-100, cyclohexane, n-hexanol and $\mathrm{HNO}_{3}$ as acid. ${ }^{22}$ From these images it can be seen that both samples consist of nanocrystallites that are roughly $3 \mathrm{~nm}$ in diameter. The particles exhibit different surface properties manifested in differences in aggregate arrangements. The sample made in the Triton X-100 microemulsion system consists of spherical aggregates with variable diameter, whereas in the sample from the Pluronic F127 microemulsion system frustum-shaped aggregates with a diameter of 30 to $50 \mathrm{~nm}$ are formed. These aggregate shapes can also be seen in the SEM pictures presented in Fig. 6.

While in solution, the properties of the two amphiphilic molecules compared differ in their ability to induce colloidal stabilization of the formed nanoparticles, which is why the subsequent particle aggregation appears different in the two microemulsion systems. In the case of Pluronic F127 its long polyethyleneoxide tails effectively adsorb to the hydrophilic $\mathrm{TiO}_{2}$ surface, while its more hydrophobic polypropyleneoxide chain extends far out in the oil matrix of the microemulsion, preventing the particles in neighboring droplets from coming in contact with each other. The much smaller polyethyleneoxide 

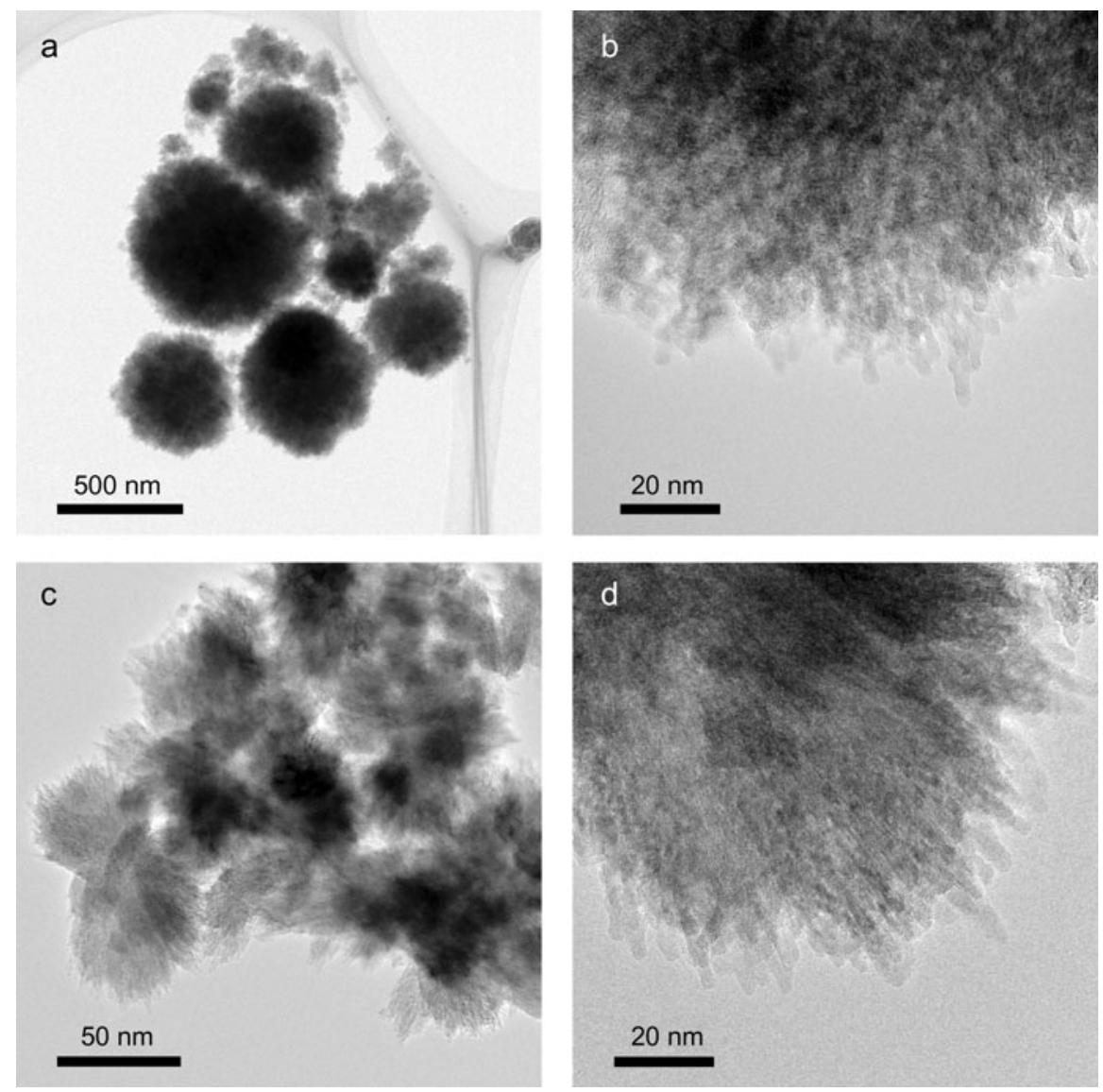

FIG. 5. TEM micrographs of a rutile sample synthesized in the Triton X-100 system ${ }^{22}$ (a) and (b) compared with a rutile sample synthesized during $8 \mathrm{~h}$ of synthesis at $40{ }^{\circ} \mathrm{C}$ using a microemulsion with composition A and $5 \mathrm{M} \mathrm{HCl}$ as acid solution (c) and (d). Spherical and frustum-shaped aggregates are found in images (a) and (c), respectively. At higher magnifications the rod-shaped crystals as well as the crystal fringes appear and differences in aggregate structures are apparent $(b, d)$.

chain of the Triton $\mathrm{X}-100$ suggests its adsorption to the $\mathrm{TiO}_{2}$ surface is lower. In addition, the short hydrophobic group of Triton X-100 extends a shorter distance in the oil matrix compared with the long hydrophobic chain of F127. The overall colloidal stabilization effect of Triton $\mathrm{X}-100$ is thus expected and found to be lower than for F127.

The structure of the nanocrystals and the manner of their aggregation were further characterized using highresolution TEM images and the corresponding Fourier diffractograms presented in Fig. 7. Both samples show reflections corresponding to rutile. The Triton X-100 sample shows a typical polycrystalline ring pattern [Fig. 7(b)]. Also, several reflections that could be expected from a powder pattern do not appear due to the preferred orientation and the alignment of the crystallites along certain crystal facets. The pattern of the Pluronic F127 sample shows single-crystal-like diffraction pattern indicative of a sample with strong preferred orientation of the nanocrystals in the aggregate. The arcs are indexed with rutile structure of [010] incidence, and some other spots are also observed from small nanocrystallites.
The reason for the differences in preferred orientation has not been investigated in detail and is thus not completely understood. In general, the resulting aggregate structure for a nanoparticle suspension will be that which is kinetically and/or energetically favored during the conditions used. Thus, it is the combination of all experimental conditions used and the surface properties of the formed particles that govern which specific aggregate structure that results. A number of key differences between the two studied microemulsion systems hold plausible explanations for the observations made including: (i) differences in adsorption strength of the amphiphile on different facets of the growing crystal leading to nonspherical particle morphologies, (ii) differences in induced surface characteristics of the nanoparticles by the adsorption of the amphiphiles, and (iii) the self-assembling properties of the amphiphile and differences in their sensitivity toward the presence of nanoparticles.

Interestingly, the crystallite size of the products formed with the Pluronic F127 microemulsion was found to be 8 to $9 \mathrm{~nm}$ for all samples prepared when determined with $\mathrm{x}$-ray line broadening. Despite the small particle size, 

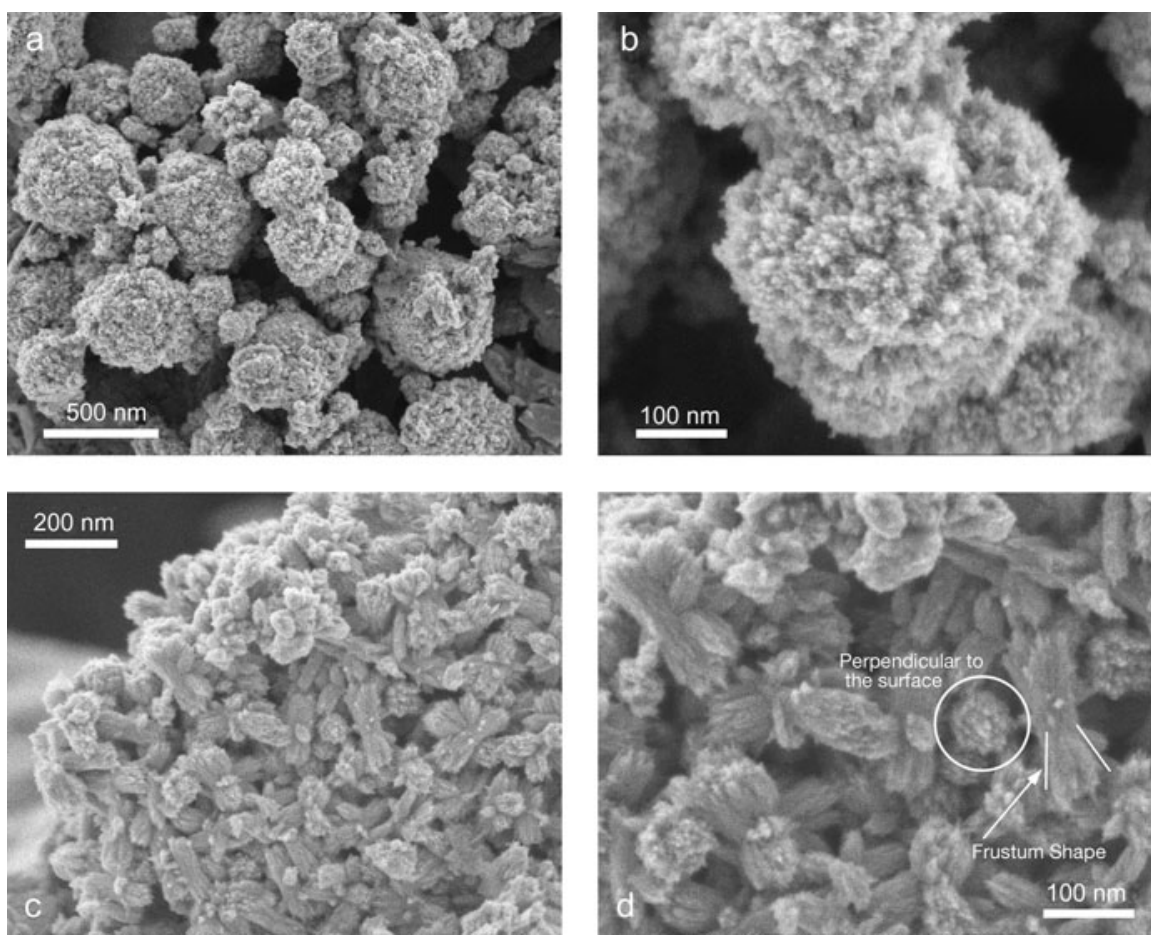

FIG. 6. SEM images of a sample synthesized in the Triton X-100 system (a) and (b) compared with a sample formed after $8 \mathrm{~h}$ of synthesis at $40{ }^{\circ} \mathrm{C}$ using a microemulsion with composition $\mathrm{A}$ and $5 \mathrm{M} \mathrm{HCl}$ as acid solution (c) and (d).
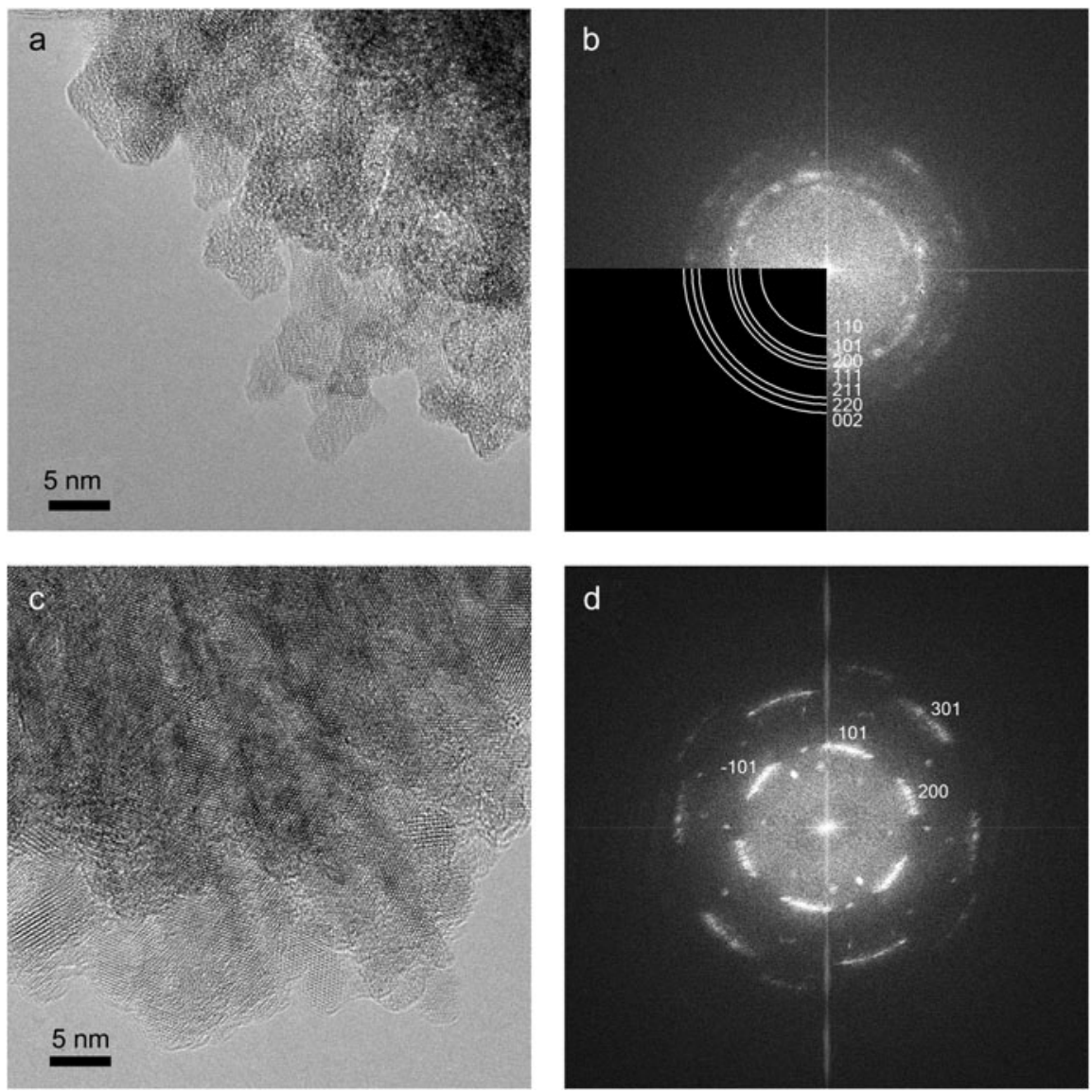

FIG. 7. High-resolution TEM images and corresponding Fourier diffractograms of samples made in the Triton X-100 microemulsion system (a) and (b) and in the Pluronic F-127 microemulsion system (c) and (d) clearly showing differences in nanoparticle sizes and their orientation relationships. 
most of these samples had a surface area below $100 \mathrm{~m}^{2} / \mathrm{g}$, except for those synthesized at $120^{\circ} \mathrm{C}$, which were close to $200 \mathrm{~m}^{2} / \mathrm{g}$. The fact that the specific surface area is not correlated with a corresponding difference in crystallite size is consistent with the strong preferred orientation observed during particle aggregation, which limits the accessible surface-to-nitrogen sorption in varying degrees. The discrepancy between the XRD and TEM crystal size measurements may also be due to the strong preferred orientation of the aggregated nanocrystallites resulting in an apparent larger crystal domain size and narrower x-ray diffraction peaks.

The results obtained show that the type of surfactant used in microemulsion-based syntheses has a large impact on the crystallization kinetics of $\mathrm{TiO}_{2}$ as they differ in several aspects compared with earlier results obtained for the Triton X-100 system. ${ }^{22}$ Especially noteworthy is that no crystallization is observed at room temperature even at longer time periods using Pluronic F127 in contrast with the Triton X-100 system. The plausible reason for these differences is the expected slower dynamics and reaction kinetics of the bulkier amphiphile system used here. In addition, the composition of the synthesis mixture within the reversed microemulsion region also has a large impact on the crystallization kinetics. At conditions with high viscosity and low water-phase content, the crystallization is very slow or absent, also expected from decreased dynamics and reaction kinetics. The influence of the anion of the acid used on the polymorph formed seems to be generic in nature. A synthesis made at hydrothermal conditions in a mixture of Triton X-100, cyclohexane and n-hexanol using $\mathrm{HCl}$ yields rutile while $\mathrm{HNO}_{3}$ gives anatase ${ }^{8,27}$ Using the Pluronic F127 system, similar results were observed at lower temperatures, where rutile is formed at $50{ }^{\circ} \mathrm{C}$ using $\mathrm{HCl}$ and anatase is formed using $\mathrm{HNO}_{3}$. The small amount of anatase present in the sample prepared using $2.5 \mathrm{M} \mathrm{HCl}$ can be understood from earlier studies that show that for a given acid anatase is favored when the $\mathrm{H}^{+} / \mathrm{Ti}$ ratio decreases. ${ }^{28}$

Since rutile is the thermodynamically stable polymorph of $\mathrm{TiO}_{2}$ and in many cases formed from an intermediate state of anatase or brookite with increasing temperature or time, ${ }^{22,29,30}$ one could expect that rutile is only formed at temperatures above $120^{\circ} \mathrm{C}$ or perhaps after longer time periods. However, there is a temperature window around $50{ }^{\circ} \mathrm{C}$ where the formation of rutile is favored. The boundary of this temperature window within the Pluronic F127 microemulsion system seems to be at 60 ${ }^{\circ} \mathrm{C}$ where both polymorphs form. There have been reports showing that anatase is favored over rutile when the crystal size is smaller than $14 \mathrm{~nm} .^{31,32}$ This is not the case in the current study, which supports instead the theory that the crystal structure thermodynamics is highly dependent on the soft wet-chemical surrounding present and offering a high mobility of species during particle formation as well as the interaction with surrounding molecular species present in the solution. ${ }^{22}$

\section{CONCLUSIONS}

A low-temperature synthesis method to prepare crystalline $\mathrm{TiO}_{2}$ nanoparticles within the Pluronic F127 microemulsion system has been developed. The synthesis route of the nanoparticles is very flexible, and both anatase and rutile polymorphs can be prepared. The aggregation properties of the prepared particles differ from those prepared in the Triton X-100 microemulsion system and result in strong preferred orientation and frustum-shaped aggregates. The development of a synthesis of nanocrystalline $\mathrm{TiO}_{2}$ in the Pluronic F127 microemulsion system enables progress to be made in methods for direct synthesis of meso-ordered $\mathrm{TiO}_{2}$ at low temperatures due to the selfassembling properties of the block copolymer.

\section{ACKNOWLEDGMENTS}

E.N. thanks the National Graduate School in Material Science and the Foundation for Strategic Research for financial support. A.E.C.P. thanks the Swedish Research Council for financial support of a Senior Researcher position. O.T. thanks V.R. and the Knut \& Alice Wallenberg Foundation for supports.

\section{SUPPORTING INFORMATION}

Powder x-ray diffractograms of materials formed at $50{ }^{\circ} \mathrm{C}$ during $96 \mathrm{~h}$ of synthesis at different positions in the $\mathrm{L}_{2}$ phase and powder $\mathrm{x}$-ray diffractograms of products formed at $50{ }^{\circ} \mathrm{C}$ of synthesis as a function of synthesis time using a microemulsion with composition $\mathrm{A}$ and $5 \mathrm{M} \mathrm{HCl}$ as acid solution.

\section{REFERENCES}

1. A. Fujishima and K. Honda: Electrochemical photolysis of water at a semiconductor electrode. Nature 238, 37 (1972).

2. M.R. Hoffmann, S.T. Martin, W.Y. Choi, and D.W. Bahnemann: Environmental applications of semiconductor photocatalysis. Chem. Rev. 95, 69 (1995).

3. A. Fujishima, T.N. Rao, and D.A. Tryk: Titanium dioxide photocatalysis. J. Photochem. Photobiol. Chem., A 1, 1 (2000).

4. B. Oregan and M. Grätzel: A low-cost, high-efficiency solar-cell based on dye-sensitized colloidal $\mathrm{TiO}_{2}$ films. Nature 353, 737 (1991).

5. Y.Z. Li, N.H. Lee, E.G. Lee, J.S. Song, and S.J. Kim: The characterization and photocatalytic properties of mesoporous rutile $\mathrm{TiO}_{2}$ powder synthesized through self-assembly of nano crystals. Chem. Phys. Lett. 389, 124 (2004).

6. S.J. Kim, H.G. Lee, S.J. Kim, J.K. Lee, E.G. Lee: Photoredox properties of ultrafine rutile $\mathrm{TiO}_{2}$ acicular powder in aqueous 4chlorophenol, Cu-EDTA and Pb-EDTA solutions. Appl. Catal., A 242, 89 (2003) 
7. J. Sun, L. Gao, and Q.H. Zhang: Synthesizing and comparing the photocatalytic properties of high surface area rutile and anatase titania nanoparticles. J. Am. Ceram. Soc. 86, 1677 (2003).

8. M. Andersson, L. Österlund, S. Ljungstrom, and A. Palmqvist: Preparation of nanosize anatase and rutile $\mathrm{TiO}_{2}$ by hydrothermal treatment of microemulsions and their activity for photocatalytic wet oxidation of phenol. J. Phys. Chem. B 106, 10674 (2002).

9. M. Andersson, H. Birkedal, N.R. Franklin, T. Ostomel, S. Boettcher, A.E.C. Palmqvist, and G.D. Stucky: Ag/AgCl-loaded mesoporous anatase for photocatalysis. Chem. Mater. 17, 1409 (2005).

10. M. Grätzel and J.R. Durrant: Dye-sensitized mesoscopic solar cells, in Nanostructured and Photoelectrochemical Systems for Solar Photon Conversion, vol. 3, edited by M.D. Archer and A.J. Nozik (Imperial College Press, London, 2008).

11. T. Yanagisawa, T. Shimizu, K. Kuroda, and C. Kato: The preparation of alkyltrimethylammonium-kanemite complexes and their conversion to microporous materials. Bull. Chem. Soc. Jpn. 63, 988 (1990).

12. C.T. Kresge, M.E. Leonowicz, W.J. Roth, J.C. Vartuli, and J.S. Beck: Ordered mesoporous molecular-sieves synthesized by a liquid-crystal template mechanism. Nature 359, 710 (1992).

13. J.S. Beck, J.C. Vartuli, W.J. Roth, M.E. Leonowicz, C.T. Kresge, K.D. Schmitt, C.T.W. Chu, D.H. Olson, E.W. Sheppard, S.B. Mccullen, J.B. Higgins, and J.L. Schlenker: A new family of mesoporous molecular-sieves prepared with liquid-crystal templates. J. Am. Chem. Soc. 114, 10834 (1992).

14. G.S. Attard, J.C. Glyde, and C.G. Göltner: Liquid-crystalline phases as templates for the synthesis of mesoporous silica. Nature 378, 366 (1995).

15. P.D. Yang, D.Y. Zhao, D.I. Margolese, B.F. Chmelka, and G.D. Stucky: Generalized syntheses of large-pore mesoporous metal oxides with semicrystalline frameworks. Nature 396, 152 (1998).

16. D.Y. Zhao, Q.S. Huo, J.L. Feng, B.F. Chmelka, and G.D. Stucky: Nonionic triblock and star diblock copolymer and oligomeric surfactant syntheses of highly ordered, hydrothermally stable, mesoporous silica structure. J. Am. Chem. Soc. 120, 6024 (1998).

17. C.J. Brinker, Y. Lu, A. Sellinger, and H. Fan: Evaporation-Induced self-assembly: Nanostructures made easy. Adv. Mater. 11, 579 (1999).

18. P.C.A. Alberius, K.L. Frindell, R.C. Hayward, E.J. Kramer, G.D. Stucky, and B.F. Chmelka: General predictive syntheses of cubic, hexagonal, and lamellar silica and titania mesostructured thin films. Chem. Mater. 14, 3284 (2002).
19. H.S. Nalwa: Nanoclusters and Nanocrystals (American Scientific Publishers, Stevenson Ranch, CA, 2003),

20. K.J. Klabunde: Nanoscale Materials in Chemistry (WileyInterscience, New York, 2001)

21. P. Hald, J. Becker, M. Bremholm, J.S. Pedersen, J. Chevallier, S.B. Iversen, and B.B. Iversen: Supercritical propanol-water synthesis and comprehensive size characterisation of highly crystalline anatase $\mathrm{TiO}_{2}$ nanoparticles. J. Solid State Chem. 179, 2674 (2006).

22. M. Andersson, A. Kiselev, L. Österlund, and A.E.C. Palmqvist: Microemulsion-mediated room-temperature synthesis of high surface area rutile and its photocatalytic performance. J. Phys. Chem. C 111, 6789 (2007).

23. P. Holmqvist, P. Alexandridis, and B. Lindman: Modification of the microstructure in block copolymer-water-"oil" systems by varying the copolymer composition and the "oil" type: Small-angle x-ray scattering and deuterium-NMR investigation. J. Phys. Chem. B 102, 1149 (1998).

24. P. Scherrer: Bestimmung der Grösse und der inneren Struktur von Kolloidteilchen mittels Röntgenstrahlen. Göttinger Nachricten. 2, 98 (1918).

25. A.L. Patterson: The Scherrer formula for $x$-ray particle size determination. Phys. Rev. 56, 978 (1939).

26. S. Brunauer, P.H. Emmett, and E.J. Teller: Adsorption of gases in multimolecular layers. J. Am. Chem. Soc. 60, 309 (1938).

27. M.M. Wu, J.B. Long, A.H. Huang, Y.J. Luo, S.H. Feng, and R.R. $\mathrm{Xu}$ : Microemulsion-mediated hydrothermal synthesis and characterization of nanosize rutile and anatase particles. Langmuir $\mathbf{1 5}$, 8822 (1999).

28. S. Yuan, Q.R. Sheng, J.L. Zhang, F. Chen, and M. Anpo: The roles of acid in the synthesis of mesoporous titania with bicrystalline structure. Mater. Lett. 58, 2757 (2004).

29. J.G. Yu, J.C. Yu, M.K.P. Leung, W.K. Ho, B. Cheng, X.J. Zhao, and J.C. Zhao: Effects of acidic and basic hydrolysis catalysts on the photocatalytic activity and microstructures of bimodal mesoporous titania. J. Catal. 217, 69 (2003).

30. J.G. Yu, J.C. Yu, B. Cheng, S.K. Hark, and K. Iu: The effect of $\mathrm{F}^{-}$doping and temperature on the structural and textural evolution of mesoporous $\mathrm{TiO}_{2}$ powders. J. Solid State Chem. 174, 372 (2003).

31. H.Z. Zhang and J.F. Banfield: Thermodynamic analysis of phase stability of nanocrystalline titania. J. Mater. Chem. 8, 2073 (1998).

32. H.Z. Zhang and J.F. Banfield: Understanding polymorphic phase transformation behavior during growth of nanocrystalline aggregates: Insights from $\mathrm{TiO}_{2}$. J. Phys. Chem. B 104, 3481 (2000). 